\section{The placebo response and pain: understanding mechanisms and their importance in pain medicine}

\section{Jo Murrell}

The placebo response can be defined as a 'a change in a patient's illness attributed to the symbolic import of a sham treatment perceived by the patient rather than a specific pharmacological or physiologic property'. However, in animals, the typical placebo effect experienced by humans is unlikely, given that it is difficult to conceive that animals have an understanding of the beneficial effects of a treatment such as an analgesic drug being given to them. Instead two different placebo effects have been well described: a caregiver placebo effect and a placebo by proxy effect.

The caregiver placebo effect was first defined in veterinary medicine by Conzemius and Evans in 2012 as 'a sham medical intervention that causes pet caregivers (owners or veterinarians) to believe the treatment they provided to the pet improved the pet's condition'. In the caregiver placebo effect there is an improvement in subjective outcome measures such as clinical metrology instruments completed by the owners in pain studies, whereas there is no accompanying improvement in objective measures such as in outcome measures from gait analysis. The caregiver placebo effect has been clearly demonstrated in randomized placebo controlled studies investigating analgesic efficacy of non-steroidal antiinflammatory drugs in dogs with osteoarthritis and cats.

The placebo by proxy effect has been well recognized in human medicine for a number of years and has been suggested to occur in clinical pain trials in animals. This is when improvements in objective outcome measures are documented in the placebo arm of the trial and are attributed to a change in the way that the owner or veterinary surgeon interacts with the animal. For example, due to a belief that the placebo is an active treatment in a blind trial the owner may walk a dog for further each day which will influence objective accelerometry data.

Placebo effects are very important in clinical research trials because the magnitude of the placebo effect may be sufficient to mask the effects of the active treatment, such that there is no statistically significant difference between placebo and treatment with improvements in both groups. Placebo effects are also likely to influence owner-perceived reports of drug effect in clinical patients which may make it difficult to judge whether an analgesic treatment is efficacious or not.

\section{KEY LEARNING OBJECTIVES}

- Know the mechanisms and factors influencing the placebo and nocebo effect in man

- Know the different placebo effects in veterinary medicine

- Know how the placebo effect influences analgesic clinical research trials

\section{MULTIPLE CHOICE QUESTIONS}

1. What are the effects of the caregiver placebo response in a clinical research trial?

(A) An improvement in subjective outcome measures with no change in objective outcome measures in the placebo group

(B) An improvement in both subjective and objective outcome measures in the placebo group

(C) An improvement in objective outcome measures with no change in subjective outcome measures in the placebo group

(D) A deterioration in subjective outcome measures with no change in objective outcome measures in the placebo group

2. What augments the placebo response in man?

(A) The administration of smaller tablets

(B) The administration of foul-tasting medication

(C) A clinician that treats the patient without empathy

(D) The patient is given information that the medicine may have negative side effects

3. Why is the placebo response important in analgesia clinical research trials?

(A) Having placebo responders is important to ensure a heterogeneous population

(B) It helps to ensure that the study is adequately blinded

(C) It can obscure the efficacy of the test treatment

(D) It falsely increases the efficacy of the test treatment 